\title{
Analysis of the Effect of Interference on InSAR
}

\author{
Huaping Xu, Member, IEEE, Zhefeng Wu, Wei Liu, Senior Member, IEEE, Jingwen Li, and Qingqing Feng
}

\begin{abstract}
Various jamming techniques have been developed to prevent interferometric synthetic aperture radar from effective detection and observation. In this paper, a thorough analysis of the jamming effects on correlation and interferometric phase is provided. To derive the jamming result, a general signal model for the interference is first presented and the corresponding imaging results are produced through the range-Doppler algorithm. Then, the impacts of the interference on correlation are analyzed. The non-center located jammer decreases the correlation seriously due to the low correlation of the interference. However, the center located jammer clearly increases the correlation when the input jamming-to-signal ratio is large enough. Finally, the jammed interferometric phases for different jammer positions are discussed. It shows that the non-center located jammer results in large phase errors, while for the center located jammer, the interferometric phase approaches a constant. The effects of interference are demonstrated by simulated data based on the TerraSAR system.
\end{abstract}

Index Terms-SAR, interfereometry, interference, correlation, jamming to signal ratio.

\section{INTRODUCTION}

A S AN all-weather all-time remote sensing technique, the synthetic aperture radar (SAR) has been widely applied to areas such as monitoring, geological investigation and ground moving target recognition, and therefore is of great importance in both military and civilian applications [1]. InSAR, a further development of the traditional SAR technology, employs two SAR images of the same terrain derived from the master antenna and the slave antenna respectively to obtain the elevation information of the ground surface through interferometry [2].

In the past, some InSAR systems have been deployed for different purposes. The satellite TerraSAR-X launched in late 2006 provides high-resolution ocean current measurements by along-track InSAR in various experimental modes of operation [3]. TanDEM-X, TerraSAR-X's twin satellite, has the mission of deriving a global digital elevation model (DEM)

Manuscript received February 11, 2015; revised May 12, 2015; accepted June 9, 2015. Date of publication June 16, 2015; date of current version August 12, 2015. This work was supported in part by the Program for New Century Excellent Talents in University, in part by the National Natural Science Foundation of China under Grant 61471020, and in part by China Aerospace Science and Technology Corporation. The associate editor coordinating the review of this paper and approving it for publication was Dr. Francis P. Hindle.

H. Xu, Z. Wu, J. Li, and Q. Feng are with the School of Electronic and Information Engineering, Beihang University, Beijing 100084, China (e-mail: xuhuaping@buaa.edu.cn; wuzheming001@126.com; lijingwen@buaa.edu.cn; 18010127731@163.com).

W. Liu is with the Department of Electronic and Electrical Engineering, University of Sheffield, Sheffield S1 3JD, U.K (e-mail: w.liu@ sheffield.ac.uk).

Color versions of one or more of the figures in this paper are available online at $\mathrm{http}: / /$ ieeexplore.ieee.org.

Digital Object Identifier 10.1109/JSEN.2015.2445931 with an unprecedented accuracy corresponding to HRTI-3 specifications [4]. The Shuttle Radar Topography Mission (SRTM), using dual-antenna and single-pass InSAR, represents a major step forward in the generation of a nearly global DEM with homogeneous coverage and accuracy [5]. All these InSAR systems have high measuring accuracy and can detect a variety of important targets effectively.

Meanwhile, due to the rapid development of electronic countermeasure (ECM), various types of jamming signals and interfering methods have been used in practice [6]-[9]. With strong electromagnetic interference, the quality of SAR images, interferogram and DEM may degrade significantly. In order to improve the robustness of an InSAR system in different environments, it is extremely important to analyze the effects of interferences on the system. Moreover, research in jamming effects can help identify the weakness of our current InSAR systems, so that more effective and robust systems can be developed in the future.

The effects of interferences on the performance of various SAR systems have already been studied [10]-[13]. Nevertheless, the relevant research for the interferometric characteristics is insufficient and incomprehensive. Bin Ding et al. analyzed the impact of RFI on point target SAR imaging and studied the effectiveness of RFI on interferometric phase through computer simulations [14]. However, it did not consider the influence of jammer positions and no theoretical analysis was provided. In [15], the phase difference of the echo for the reflected jamming signal was obtained, but it cannot be employed to directly analyze the interferometric characteristics. Liu et al. derived the interferometric phase result with an active coherent interference of arbitrary waveform modulation in [16], but the signal model adopted there does not consider the protected targets and the correlation of the corresponding pixel pairs is not analyzed.

In this paper, the jamming effects on single-pass InSAR are investigated. First, the imaging results of both antennas are produced using the presented interference signal model through the Range-Doppler algorithm. Since the interference can be partly compressed in azimuth direction, the output of interference is displayed as a jamming strip along range. Then, the correlation, employed to guide the following phase unwrapping algorithms, is derived by utilizing the pixel pairs in two SAR images. It shows that the correlation of the jammed pixel pair is determined by the input JSR and the position of the jammer. When the jammer is not located at the center of the jammed area (the non-center located jammer), the position deviation between the jammer and scene center will reduce the correlation with the increase of JSR. For the jammer which is located at the center of the jammed area 
(the center located jammer), the correlation is reduced when the power of interference is approximately equal to that of the SAR images. When the JSR is large enough, increasing JSR will improve the correlation of the pixel pair in two SAR images. Finally, the interferometric phase due to interference is calculated. It shows that the interferometric phase due to the center located jammer approaches to a constant, when the input JSR is further increased. However, the non-center located jammer results in large phase errors. All these analyses are obtained with respect to different jammer positions.

The remainder of this paper is organized as follows. Signal models and imaging outputs due to the interference are given in Section II. In Section III, the jamming effect on the correlation is analyzed, while the interferometric phase of the jammed pixel pair is derived. Simulation results are provided in Section IV and conclusions are drawn in Section V.

\section{INTERFERENCE MODEL AND SAR IMAGING RESUlT}

In this section, based on the geometry of InSAR and the jammer, a general model with interference is established and the echo signal model due to interference for single-pass InSAR is obtained. The imaging outputs of two antennas are derived through the Range-Doppler algorithm. They are used to analyze the influence of interference on interferometry.

\section{A. Mathematical Models of Interferences}

There are four widely used interference signal models in literature: radio frequency interference, amplitude-modulated interference, frequency-modulated interference, and phasemodulated interference [7]. Consider the following general expression for interference [17]:

$$
J(t)=a(t) \exp \left\{j 2 \pi f_{c} t\right\}
$$

where $f_{c}$ is the carrier frequency and $a(t)$ is given by

$$
a(t)=A(t) \exp \{j[u(t) t+\varphi(t)]\}
$$

with $A(t)$ representing amplitude of the interference, and $u(t)$ and $\varphi(t)$ denoting its baseband frequency and the phase part, respectively. All three parameters are time-varying.

\section{B. Echo Model for Interferences}

A typical scenario with ECM is shown in Fig. 1 with the InSAR geometry, a jammer and the beam footprint, where points $A_{1}$ and $A_{2}$ are the master antenna and the slave antenna, respectively. The jammer is considered as a stationary point target within one synthetic aperture period; the SAR platform flies along the y-axis at a fixed altitude with the speed of $v$ and images an area on the ground plane described by $x \in\left[X_{c}-X_{0}, X_{c}+X_{0}\right]$ and $y \in\left[-Y_{0}, Y_{0}\right] . \theta$ and $\alpha$ denote the look angle and the inclination of the baseline, respectively. $B$ is the length of the baseline, $H$ is the flight altitude of the master antenna, and $X O Y$ (ground plane) is the target focus plane. The instantaneous slant ranges between the phase center of both antennas and the jammer at $t_{a}$ are expressed as

$$
\begin{aligned}
R_{m j}\left(t_{a}\right) & =\sqrt{R_{J 1}^{2}+\left(v t_{a}\right)^{2}} \\
R_{s j}\left(t_{a}\right) & =\sqrt{R_{J 2}^{2}+\left(v t_{a}\right)^{2}}
\end{aligned}
$$

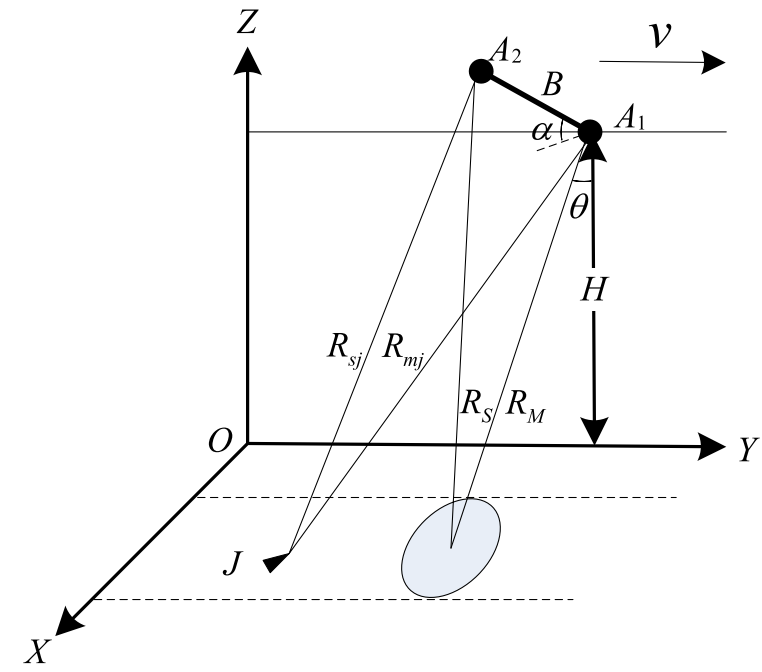

Fig. 1. InSAR and jammer geometry.

where $R_{J 1}$ and $R_{J 2}$ are the minimum slant ranges between the phase center of two antennas and jammer. If the interference is transmitted directly to the SAR antennas, the Doppler frequency of the interference will be quite different from that of the echo signal from the protected scene. To prevent the interference from being filtered out through the azimuth filter, a time delay is always added to remove the azimuth frequency difference. Suppose that the time delay of the interference is $\Delta \tau=R_{m j}\left(t_{a}\right) / c$, and both the interference and SAR echo signals have the same carrier frequency. After down-converting, the interfering signal jamming the master image can be expressed as

$$
\begin{aligned}
J_{m}\left(t_{r}, t_{a}\right)= & \operatorname{rect}\left[\frac{t_{a}}{T_{L}}\right] a\left[t_{r}-\frac{2 R_{m j}\left(t_{a}\right)}{c}\right] \\
& \times \exp \left\{-j \frac{4 \pi R_{m j}\left(t_{a}\right)}{\lambda}\right\}
\end{aligned}
$$

where $c$ is the speed of light, $T_{L}$ is the synthetic aperture time, $\lambda$ is the wavelength, $t_{r}$ and $t_{a}$ denote the fast (range) time and the slow (azimuth) time, and rect[·] is the rectangular window function.

For the single-pass InSAR, one antenna serves as the transmitter and both antennas record the scattered signal simultaneously. As a result, the interference received by the slave antenna with time delay $\Delta \tau$ can be expressed as

$$
\begin{aligned}
J_{s}\left(t_{r}, t_{a}\right)= & \operatorname{rect}\left[\frac{t_{a}}{T_{L}}\right] a\left[t_{r}-\frac{R_{m j}\left(t_{a}\right)+R_{s j}\left(t_{a}\right)}{c}\right] \\
& \cdot \exp \left\{-j \frac{2 \pi\left[R_{m j}\left(t_{a}\right)+R_{s j}\left(t_{a}\right)\right]}{\lambda}\right\}
\end{aligned}
$$

\section{Imaging Result of Interferences}

We now develop image models due to interference in the single-pass mode. There are different imaging algorithms available. Without loss of generality and for simplicity, the Range-Doppler algorithm in [18] is used to generate the SAR imaging result here. The principle of stationary phase (POSP) is applied to obtain an analytic expression of the azimuth compression of the interference. 
First, range matched filtering is performed to (5) using the filter

$$
h_{r_{r} r e f}\left(t_{r}\right)=\operatorname{rect}\left[\frac{t_{r}}{T_{P}}\right] \exp \left\{-j \pi K_{r} t_{r}^{2}\right\}
$$

where $T_{P}$ is the pulse duration, and $K_{r}$ denotes the chirp rate. The range-compressed output for the interference at the master antenna is given by

$$
\begin{aligned}
J_{m \_r c}\left(t_{r}, t_{a}\right)= & {\left[a\left(t_{r}-\frac{2 R_{m j}\left(t_{a}\right)}{c}\right) \otimes h_{r_{-} r e f}\left(t_{r}\right)\right] } \\
& \cdot \operatorname{rect}\left[\frac{t_{a}}{T_{L}}\right] \exp \left\{-j \frac{4 \pi R_{m j}\left(t_{a}\right)}{\lambda}\right\}
\end{aligned}
$$

where $\otimes$ denotes the convolution operation. The compressed interference after Range Cell Migration Correction (RCMC) and azimuth matched filtering is given by

$$
\begin{aligned}
J_{m_{-} \text {out }}\left(t_{r}, t_{a}\right)= & a^{\prime}\left[t_{r}-\frac{2 R_{J 1}}{c}\right] \cdot \int \operatorname{rect}\left[\frac{\tau}{T_{L}}\right] \operatorname{rect}\left[\frac{t_{a}-\tau}{T_{L}}\right] \\
& \times \exp \left\{j \theta_{m}\left(\tau, t_{a}\right)\right\} d \tau
\end{aligned}
$$

where $a^{\prime}[\bullet]$ represents the compression of range jamming, $R_{M}$ and $R_{S}$ are defined as the minimum slant ranges between the phase center of two antennas and the center of the scene, respectively. Then, we define the Doppler chirp rate of the master image as $K_{a 1}=2 v^{2} /\left(\lambda R_{M}\right)$. In (9), we have

$$
\theta_{m}\left(\tau, t_{a}\right)=-\frac{4 \pi R_{m j}(\tau)}{\lambda}+\pi K_{a 1}\left(t_{a}-\tau\right)^{2}
$$

By utilizing the POSP with the stationary point $\tau=t_{a} /\left(1-\xi_{1}\right)$ and $\xi_{1}=R_{M} / R_{J 1}$, the imaging output for the interference at the master antenna changes to

$$
\begin{aligned}
J_{m_{-} \text {out }}\left(t_{r}, t_{a}\right) & \\
= & \frac{1}{\sqrt{K_{a 1}\left|1-\xi_{1}\right|}} \operatorname{rect}\left[\frac{t_{a}}{\left(1-\xi_{1}\right) T_{L}}\right] a^{\prime}\left[t_{r}-\frac{2 R_{J 1}}{c}\right] \\
& \cdot \exp \left\{j \pi K_{a 1} \frac{\xi_{1}}{\xi_{1}-1} t_{a}^{2}\right\} \exp \left\{-j \frac{4 \pi R_{J 1}}{\lambda}\right\}
\end{aligned}
$$

Similar to (11), we obtain the imaging result of the interference at the slave antenna as

$$
\begin{aligned}
J_{S_{-} \text {out }}\left(t_{r}, t_{a}\right) & \frac{1}{\sqrt{K_{a 2}\left|1-\xi_{2}\right|}} \operatorname{rect}\left[\frac{t_{a}}{\left(1-\xi_{2}\right) T_{L}}\right] a^{\prime}\left[t_{r}-\frac{R_{J 1}+R_{J 2}}{c}\right] \\
& \cdot \exp \left\{j \pi K_{a 2} \frac{\xi_{2}}{\xi_{2}-1} t_{a}^{2}\right\} \exp \left\{-j \frac{2 \pi\left(R_{J 1}+R_{J 2}\right)}{\lambda}\right\}
\end{aligned}
$$

with

$$
\left\{\begin{array}{l}
K_{a 2}=\frac{v^{2}}{\lambda}\left(\frac{1}{R_{M}}+\frac{1}{R_{S}}\right) \\
\xi_{2}=\frac{1 / R_{J 1}+1 / R_{J 2}}{1 / R_{M}+1 / R_{S}}
\end{array}\right.
$$

When the jammer is located in the center of the scene, we have $R_{m j}\left(t_{a}\right)=R_{m}\left(t_{a}\right)$ and $R_{s j}\left(t_{a}\right)=R_{s}\left(t_{a}\right)$, where $R_{m}\left(t_{a}\right)$ and $R_{S}\left(t_{a}\right)$ represent the instantaneous slant ranges between the phase center of both antennas and the center of the scene. The interference along azimuth is completely compressed after
TABLE I

SIMULATION PARAMETERS OF THE InSAR SYSTEM

\begin{tabular}{cc}
\hline \hline Parameter & Value \\
\hline Carrier frequency & $9.6 \mathrm{GHz}$ \\
Chirp rate & $1.3 \times 10^{13} \mathrm{~Hz} / \mathrm{s}$ \\
Pulse duration & $10 \mu \mathrm{s}$ \\
Sampling frequency & $145 \mathrm{MHz}$ \\
Sensor velocity & $7604 \mathrm{~m} / \mathrm{s}$ \\
Squint angle & $0^{\circ}$ \\
Center squint range & $545.1 \mathrm{~km}$ \\
Altitude & $514.8 \mathrm{~km}$ \\
Baseline length & $200 \mathrm{~m}$ \\
Baseline inclination & $0^{\circ}$ \\
\hline \hline
\end{tabular}

2D matched filtering. Therefore, the imaging output for the interference in the master image becomes

$$
J_{m \_o u t}\left(t_{r}, t_{a}\right)=T_{L} \cdot \operatorname{sinc}\left(t_{a}\right) a^{\prime}\left[t_{r}-\frac{2 R_{M}}{c}\right] \exp \left\{-j \frac{4 \pi R_{M}}{\lambda}\right\}
$$

Similarly, the interference in the slave image can be expressed as

$$
\begin{aligned}
J_{S_{-} \text {out }}\left(t_{r}, t_{a}\right)= & T_{L} \cdot \operatorname{sinc}\left(t_{a}\right) a^{\prime}\left[t_{r}-\frac{2\left(R_{M}+R_{S}\right)}{c}\right] \\
& \times \exp \left\{-j \frac{2 \pi\left(R_{M}+R_{S}\right)}{\lambda}\right\}
\end{aligned}
$$

Clearly, in either case, the received interference can be compressed to some extent. In the range dimension, the compression depends on the correlation between baseband signal of the interference and range matched filtering. However, the azimuth compression is related to jammer positions and the Doppler frequency.

\section{EFFECT OF INTERFERENCES ON InSAR}

Based on signal models derived in Section II, we now analyze the interfering effects on the InSAR system. First, the correlation of the pixel pair due to the interference is derived, followed by the resultant interferometric phase. Both theoretical analyses and simulation results are provided in this section, with simulation parameters listed in Table I, unless otherwise noted.

\section{A. Correlation of InSAR Image Pair With Interference}

Correlation, a measure of similarity between two single-look complex (SLC) images, provides a quantitative measure of surface and subsurface scattering properties [19], [20], and its value has a significant effect on the following interferometric phase estimation result.

Mathematically, the correlation $\gamma$ between two images $S_{1}$ and $S_{2}$ is defined as

$$
\gamma=\frac{\left|\left\langle S_{1} \cdot S_{2}^{*}\right\rangle\right|}{\sqrt{\left\langle S_{1} \cdot S_{1}^{*}\right\rangle\left\langle S_{2} \cdot S_{2}^{*}\right\rangle}}
$$


where $(\cdot)^{*}$ denotes complex conjugate, $\langle\cdot\rangle$ represents ensemble averaging, and $|\cdot|$ is the absolute value. Then, the pixel in two SAR images with interference can be expressed as

$$
\begin{aligned}
& S_{1}=A \cdot S_{\text {real_m }}+A_{j} \cdot J_{m_{-} \text {out }} \\
& S_{2}=A \cdot S_{\text {real_s }}+A_{j} \cdot J_{S_{-} \text {out }}
\end{aligned}
$$

where $S_{\text {real_m }}$ and $S_{\text {real_s }}$ represent the pixel pair before jamming with the correlation value of $\gamma_{0}, A$ and $A_{j}$ represent the magnitude of transmitted signal and input interference. The average power of the conjugate multiplication of corresponding pixel pair is given by

$$
\begin{aligned}
& \left\langle S_{1} \cdot S_{2}^{*}\right\rangle \\
& =\left\langle\left(A \cdot S_{\text {real_m }}+A_{j} \cdot J_{m \_o u t}\right) \cdot\left(A \cdot S_{\text {real_s }}+A_{j} \cdot J_{s \_o u t}\right)^{*}\right\rangle a \\
& =A^{2}\left\langle S_{\text {real_m }} \cdot S_{\text {real_s }}^{*}\right\rangle+A_{j}^{2}\left\langle J_{m \_o u t} \cdot J_{s_{-} \text {out }}^{*}\right\rangle
\end{aligned}
$$

Now, we consider the impact of jammer position on the correlation value between the jammed pixel pair. Detailed analyses are provided as follows.

1) Interference From Non-Center Located Jammer: With this condition, the individual signal powers of the jammed pixels in two images are similar to each other and they are given by

$$
\begin{aligned}
\left\langle S_{1} \cdot S_{1}^{*}\right\rangle & =A^{2}\left\langle S_{\text {real_m }} \cdot S_{\text {real_m }}^{*}\right\rangle+A_{j}^{2}\left\langle J_{m_{-} \text {out }} \cdot J_{m_{-} \text {out }}^{*}\right\rangle \\
& =A^{2}\left(T_{P} T_{L}\right)^{2}+A_{j}^{2} \frac{1}{K_{a 1}\left|1-\xi_{1}\right|} \\
\left\langle S_{2} \cdot S_{2}^{*}\right\rangle & =A^{2}\left\langle S_{\text {real_s }} \cdot S_{\text {real_s }}^{*}\right\rangle+A_{j}^{2}\left\langle J_{S_{-} \text {out }} \cdot J_{S_{-} \text {out }}^{*}\right\rangle \\
& =A^{2}\left(T_{P} T_{L}\right)^{2}+A_{j}^{2} \frac{1}{K_{a 2}\left|1-\xi_{2}\right|}
\end{aligned}
$$

According to (16), the average value of conjugate multiplication for the pixel pair without interference can be expressed as

$$
\left\langle S_{\text {real_m }} \cdot S_{\text {real_s }}^{*}\right\rangle=\left(T_{P} T_{L}\right)^{2} \gamma_{0} \exp \{j \varphi\}
$$

where $\varphi$ denotes the interferometric phase of the original pixel pair. Similarly, the average value of conjugate multiplication for jamming outputs can be written as

$$
\left\langle J_{m_{-} \text {out }} \cdot J_{s_{-} \text {out }}^{*}\right\rangle=\frac{1}{\sqrt{K_{a 1} K_{a 2}\left|1-\xi_{1}\right|\left|1-\xi_{2}\right|}} \gamma_{J} \cdot \exp \left\{j \varphi_{J}\right\}
$$

where $\varphi_{J}$ is the phase difference caused by the interference. Substituting (19)-(23) into the definition (16), the correlation of the pixel pair with interference becomes

$$
\begin{aligned}
& \gamma_{1} \\
& \approx \frac{\left|\left(T_{P} T_{L}\right)^{2}\left(K_{a 1}\left|1-\xi_{1}\right|\right) \gamma_{0} \exp \{j \varphi\}+J S R \cdot \gamma_{J} \exp \left\{j \varphi_{J}\right\}\right|}{\left(T_{P} T_{L}\right)^{2}\left(K_{a 1}\left|1-\xi_{1}\right|\right)+J S R} \\
& =\frac{\left|G^{2} \gamma_{0} \exp \{j \varphi\}+J S R \cdot \gamma_{J} \exp \left\{j \varphi_{J}\right\}\right|}{G^{2}+J S R}
\end{aligned}
$$

where $G$ represents signal to jamming compression gain ratio, and $J S R=A_{j}^{2} / A^{2}$ denotes the input jamming to signal ratio.

Generally, for the single-pass InSAR, the discrepancy between $t_{r}-2 R_{M} / c$ and $t_{r}-\left(R_{M}+R_{S}\right) / c$ in two SAR images will cause an envelope shift between $S_{\text {real_m }}$ and $S_{\text {real_s}}$.
This difference can be compensated by high precision image co-registration processing. Provided that the slave image is adjusted in the light of master image, the interference in the slave image is rearranged as

$$
\begin{aligned}
J_{s}\left(t_{r}, t_{a}\right) & \frac{1}{\sqrt{K_{a 2}\left|1-\xi_{2}\right|}} \operatorname{rect}\left[\frac{t_{a}}{\left(1-\xi_{2}\right) T_{L}}\right] \\
& \times a^{\prime}\left[t_{r}-\frac{R_{J 1}+R_{J 2}}{c}-\frac{R_{M}-R_{S}}{c}\right] \\
& \cdot \exp \left\{j \pi K_{a 2} \frac{\xi_{2}}{\xi_{2}-1} t_{a}^{2}\right\} \exp \left\{-j \frac{2 \pi\left(R_{J 1}+R_{J 2}\right)}{\lambda}\right\}
\end{aligned}
$$

For the non-center located jammer, we have

$$
\frac{R_{J 1}+R_{J 2}}{c}+\frac{R_{M}-R_{S}}{c} \neq \frac{2 R_{J 1}}{c}
$$

From (11) and (25), we can see that the time delay for $a^{\prime}\left(t_{r}\right)$ in the two equations is different. Since the interference is independent at different time, the interferences in two SAR images are independent of each other after co-registration. In this case, the correlation of the interferences is far less than that of the original pixel pair. Therefore, we have $\gamma_{J} \ll \gamma_{0}$. The correlation of the jammed pixel pair approaches to the following results under different conditions.

a) As $J S R \ll G^{2}$, we have $J S R / G^{2} \approx 0$. According to (24), the correlation of the jammed SAR images is mainly determined by that of the corresponding pixel pair. Thus, (24) changes to

$$
\begin{aligned}
\gamma_{1} & \approx \frac{\left|\gamma_{0} \exp \{j \varphi\}+\left(J S R / G^{2}\right) \cdot \gamma_{J} \exp \left\{j \varphi_{J}\right\}\right|}{1+\left(J S R / G^{2}\right)} \\
& \approx\left|\gamma_{0} \exp \{j \varphi\}\right|=\gamma_{0}
\end{aligned}
$$

b) As $J S R \approx G^{2},(24)$ is simplified as

$$
\gamma_{1} \approx \frac{\left|\gamma_{0} \exp \{j \varphi\}+\gamma_{J} \exp \left\{j \varphi_{J}\right\}\right|}{2} .
$$

From (28), the correlation of the jammed pixel pair is mainly determined by $\gamma_{0}, \gamma_{J}, \varphi$ and $\varphi_{J}$. The difference between $\varphi$ and $\varphi_{J}$ reduces the correlation. When $\gamma_{J} \ll \gamma_{0}$, such a decreasing trend in correlation will get worse.

c) As $J S R \gg G^{2}$, we have $G^{2} / J S R \approx 0$. Then, (24) changes to

$$
\begin{aligned}
\gamma_{1} & \approx \frac{\left|\left(G^{2} / J S R\right) \cdot \gamma_{0} \exp \{j \varphi\}+\gamma_{J} \exp \left\{j \varphi_{J}\right\}\right|}{\left(G^{2} / J S R\right)+1} \\
& \approx\left|\gamma_{J} \exp \left\{j \varphi_{J}\right\}\right|=\gamma_{J}
\end{aligned}
$$

Therefore, the correlation of the jammed pixel pair can be approximated as $\gamma_{J}$.

Clearly, the correlation of the jammed pixel pair is determined by both $J S R$ and $G^{2}$. A non-center located jammer causes poor match of the interferences, and reduces correlation of the two SLC images. The correlation of the pixel pair will be lower as the input JSR increases. In this case, the interference will increase the co-registration error, which in turn causes a large phase error. 


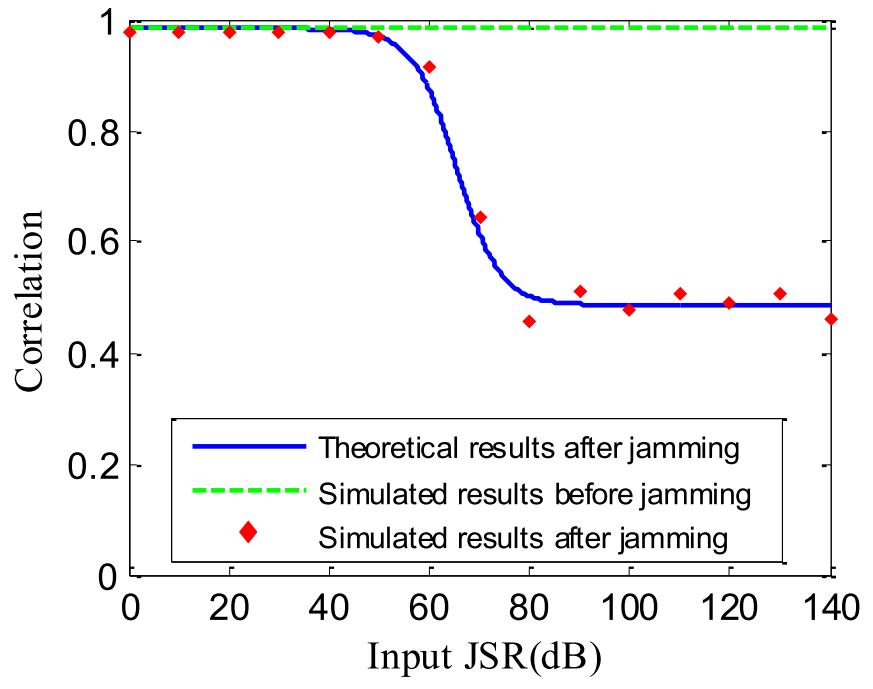

Fig. 2. Jamming effect on correlation due to the non-center located jammer.

According to the parameters listed in Table I, a ground area is established in our simulation. To analyze the jamming effect on correlation due to a non-center located jammer, correlation values with different input JSR are obtained, and the results are shown in Fig. 2, where the correlation value without interference is 0.983940 . When the input JSR is less than $50 \mathrm{~dB}$, the correlation value in this case is slightly smaller than that without jamming. When the input JSR is further increased, the correlation value is significantly reduced and finally, the correlation of the jammed pixel pair approaches that of the interference.

The solid curve in Fig. 2 is the theoretical value obtained using equation (24), in which $G^{2}$ is equal to $65.4362 \mathrm{~dB}$. When the value of the input JSR is far less than $G^{2}$, the correlation approaches to that without jamming. The increasing input JSR decreases the correlation value significantly. Ultimately, the correlation of the jammed pixel pair is approximated as that of the interference. As shown, the trend of the theoretical value is the same as that of the simulated data. Due to the varying phase difference caused by the interference, those discrete points slightly deviate from the theoretical curve. It again demonstrates the validity of the theoretical analysis.

2) Interference From Center Located Jammer: According to (15), the interference in the slave image is rearranged as

$$
\begin{aligned}
J_{S_{-} \text {out }}\left(t_{r}, t_{a}\right)= & T_{L} \cdot \operatorname{sinc}\left(t_{a}\right) a^{\prime}\left[t_{r}-\frac{2 R_{M}}{c}\right] \\
& \times \exp \left\{-j \frac{2 \pi\left(R_{M}+R_{S}\right)}{\lambda}\right\}
\end{aligned}
$$

The average value of conjugate multiplication for jamming outputs can be written as

$$
\left\langle J_{m_{-} \text {out }} \cdot J_{S_{-} \text {out }}^{*}\right\rangle=T_{L}^{2} \gamma_{J} \cdot \exp \left\{j \varphi_{J}\right\}
$$

Then, the correlation of the pixel pair with interference becomes

$$
\begin{aligned}
\gamma_{2} & =\frac{\left|\left(T_{P} T_{L}\right)^{2} \gamma_{0} \exp \{j \varphi\}+\left(T_{L}\right)^{2} J S R \cdot \gamma_{J} \exp \left\{j \varphi_{J}\right\}\right|}{\left(T_{P} T_{L}\right)^{2}+\left(T_{L}\right)^{2} J S R} \\
& =\frac{\left|T_{P}^{2} \gamma_{0} \exp \{j \varphi\}+J S R \cdot \gamma_{J} \exp \left\{j \varphi_{J}\right\}\right|}{T_{P}^{2}+J S R}
\end{aligned}
$$

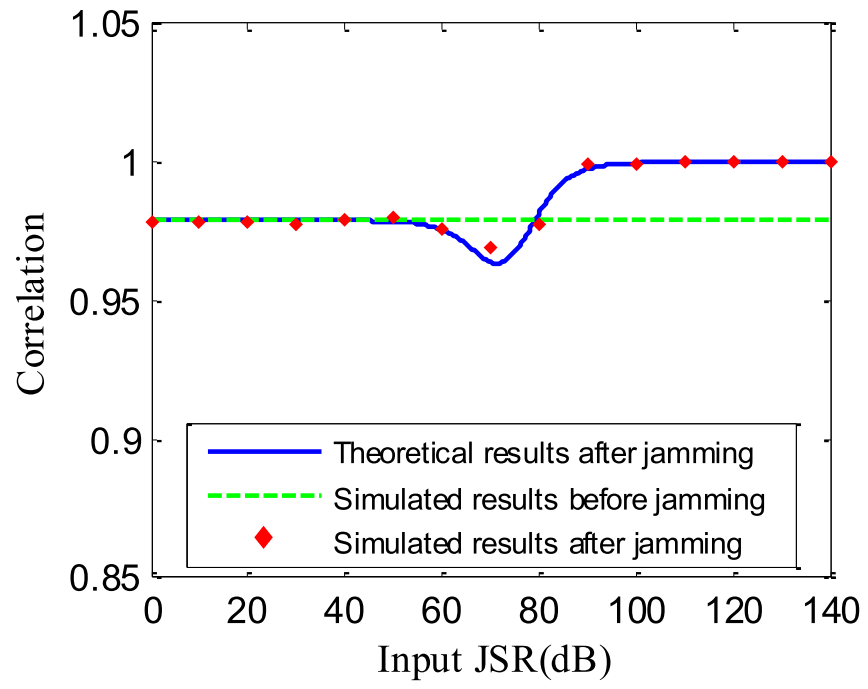

Fig. 3. Jamming effect on correlation due to the center located jammer.

Clearly, the interference in the two SAR images can be completely co-registered. Therefore, we have $\gamma_{J} \approx 1$. The correlation of the jammed pixel pair approaches to the following results under different conditions.

a) When $J S R \ll T_{P}^{2}$, the correlation of the jammed SAR images is mainly determined by that of the corresponding pixel pair. Thus, we have $\gamma_{2} \approx \gamma_{0}$.

b) When $J S R \approx T_{P}^{2}$, according to (32), we have $\gamma_{2}=\left|\gamma_{0} \exp \{j \varphi\}+\gamma_{J} \exp \left\{j \varphi_{J}\right\}\right| / 2$. The correlation of the jammed pixel pair is determined by $\gamma_{0}, \gamma_{J}, \varphi$ and $\varphi_{J}$, simultaneously. Consider the difference between $\varphi$ and $\varphi_{J}$, we have $\gamma_{2} \leq \gamma_{0}$.

c) When $J S R \gg T_{P}^{2}$, the correlation is approximated as $\gamma_{2} \approx \gamma_{J}$. Thus, the interference with large input JSR will increase the correlation of the corresponding pixel pairs and reduce the co-registration errors.

Fig.3 shows the jamming effect on correlation due to the center located jammer, with corresponding parameters listed in Table I. Clearly, as the input JSR is less than $50 \mathrm{~dB}$, the correlation for those discrete points is approximately equal to that without jamming. When the input JSR is between $50 \mathrm{~dB}$ and $80 \mathrm{~dB}$, the correlation value, due to the difference between $\varphi$ and $\varphi_{J}$, is smaller than that of the unjammed ones. As the input JSR is larger than $80 \mathrm{~dB}$, the great input JSR improves the correlation of the corresponding pixel pairs. When the input JSR is large enough, the correlation is approximated as 1 . To demonstrate the validity of the analysis results, the theoretical curves derived using (32) is shown in Fig. 3 by the solid curve. As can be seen, the theoretical results for the center-located jammer match the simulated ones very well.

By comparing Fig. 2 and Fig. 3, we can conclude that the jammer position has a clear effect on the correlation value between the corresponding pixel pairs. With further increase of the input JSR, the center located jammer increases the correlation value, while the non-center located one has an opposite effect. It has again demonstrated the effectiveness of our analysis. 


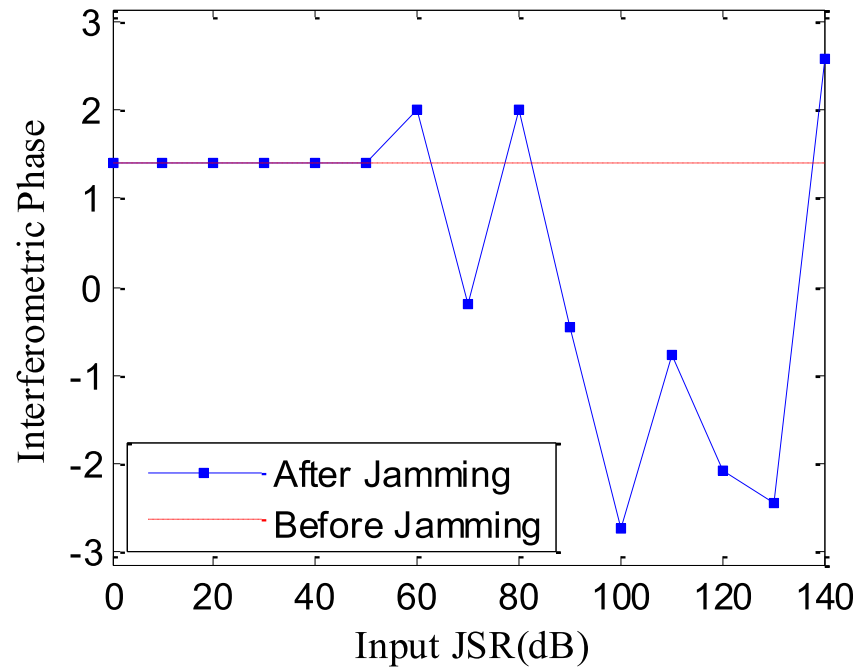

Fig. 4. Interferometric phase of the jammed pixel pair due to a non-center located jammer.

\section{B. Interferometric Phase Caused by Interference}

Interferometric phase is the basis of elevation measurement and directly affects the accuracy of DEM [21], [22]. If two SLC images are accurately co-registered, the phase difference with the interference can be obtained through conjugate multiplication of two co-registered SAR images.

1) Interference From Non-Center Located Jammer: Since the interference generates phase noise in the interferogram, different input JSR will introduce different phase errors. The interferometric phases under different conditions are considered as follows.

a) When $J S R \ll G^{2}$, the interferometric phase $\varphi_{1}$ of the corresponding pixel pair is mainly determined by the original interferometric phase $\varphi$. Thus, we have $\varphi_{1} \approx \varphi$

b) When $J S R \approx G^{2}$, the interferometric phase of the jammed pixel pair is determined by the SAR signal and interference simultaneously. Therefore, the resultant phase contains complicated phase noise.

c) When $J S R \gg G^{2}$, the interferometric phase of the jammed pixel pair due to the non-center jammer is obtained by comparing the phase of (11) and (25), given by

$$
\begin{aligned}
\varphi_{1} & =\varphi_{J} \\
& =n+\left(K_{a 1} \frac{\xi_{1}}{\xi_{1}-1}-K_{a 2} \frac{\xi_{2}}{\xi_{2}-1}\right) t_{a}^{2}-\frac{2 \pi\left(R_{J 1}-R_{J 2}\right)}{\lambda}
\end{aligned}
$$

Note that the first term $n$, due to $a^{\prime}(\cdot)$, represents the random noise. The last two terms are caused by the incomplete compression along azimuth. As can be seen, some amount of phase noise is produced when the input JSR is large enough.

Using the parameters listed in Table I, the interferometric phase of the jammed pixel pair is shown in Fig. 4. Clearly, when the input JSR is less than $50 \mathrm{~dB}$, the interferometric phase of the jammed pixel pair is approximately equal to that of the unjammed ones. This corresponds to the above analysis in the case of a). When the input JSR is further increased, large phase noises are introduced. It corresponds to

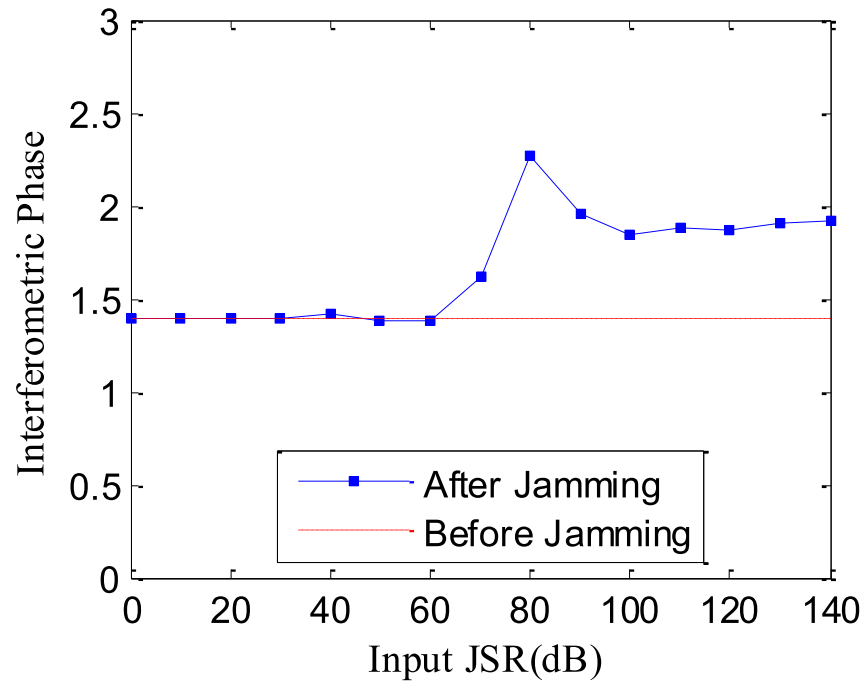

Fig. 5. Interferometric phase of the jammed pixel pair due to a center located jammer.

the result in (33). Clearly, the variation of the interferometric phase is in accordance with the theoretical analysis.

2) Interference From Center Located Jammer: The interferometric phase due to the center-located jammer approaches to the following results under different conditions.

a) When $J S R \ll T_{P}^{2}$ and $J S R \approx T_{P}^{2}$, the interferometric phase is similar to those caused by the non-centered jammer.

b) When $J S R \gg T_{P}^{2}$, the phase noise caused by $a^{\prime}(\cdot)$ is completely cancelled through conjugate multiplication. According to (14) and (15) and reference [23], we obtain the interferometric phase of the jammed pixel pair as

$$
\varphi_{2} \approx \varphi_{J}=-\frac{2 \pi\left(R_{J 1}-R_{J 2}\right)}{\lambda}
$$

Therefore, the phase noise of the jammed pixel pair approaches a constant when the input JSR is large enough.

We calculated the interferometric phase of the jammed pixel pair due to a center located jammer, and the results are shown in Fig. 5. When the input JSR is less than $60 \mathrm{~dB}$, the interferometric phase is approximately equal to the unjammed one. This is the case of $J S R \ll T_{P}^{2}$. When the input JSR is between $60 \mathrm{~dB}$ and $90 \mathrm{~dB}$, large phase errors are induced. The interferometric phase is determined by the SAR signal and interference simultaneously. It is consistent with the special case of $J S R \approx T_{P}^{2}$. When the input JSR is larger than $90 \mathrm{~dB}$, the interferometric phase can be considered as a constant, which corresponds to (34).

From Figs. 4 and 5, we see that both the jammer position and input JSR affect the interferometric phase of the jammed pixel pair. When the input JSR is large enough, the interferometric phase due to the center located jammer approaches a constant, while the one due to the non-center located jammer is shown as varying phase noise.

\section{Simulation Results and Discussion}

In this section, simulation results are provided based on the TerraSAR system to show the jamming effects. Radio frequency interference is used in our simulation. 
TABLE II

Simulation PARAmeter of THE SCENE

\begin{tabular}{cc}
\hline \hline Parameter & Value \\
\hline Range point number & 2048 \\
Azimuth point number & 2048 \\
JSR & $80 \mathrm{~dB}$ \\
\hline \hline
\end{tabular}

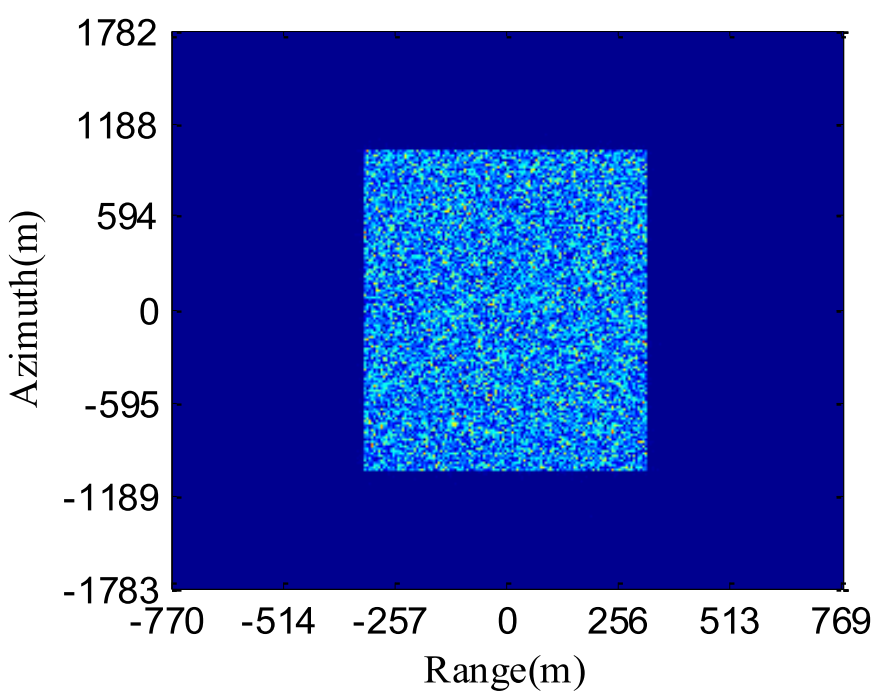

Fig. 6. The original slave images before jamming.

\section{A. Jamming Effect on Imaging}

To show the effect of interference on SAR images, we consider a flat terrain with an area of $640 \mathrm{~m} \times 2050 \mathrm{~m}$ in ground range and azimuth directions, respectively. Parameters for the system and the scene are listed in Tables I and II, respectively. Without loss of generality, only slave images are provided in this section.

For comparison purposes, the original SAR image without interference is shown in Fig. 6. Since real echo signals and the interference are received by the antennas simultaneously, simulation of the SAR echo signals with interference is first performed. JSR is set to be $80 \mathrm{~dB}$ and the positions for the non-center located jammer and the center located jammer are respectively $(500,0,0)$ and $(0,0,0)$. The slave images for different jammer positions are shown in Fig. 7. Clearly, the interferences in the two slave images are compressed to some extent along azimuth. In the range direction, the defocused interferences are caused by the low correlation value between baseband signal of the interference and range matched filtering, which are given in equations (12) and (15).

\section{B. Jamming Effect on Correlation}

As shown in the first sub-section, the slave images for different jammer positions are similar with each other. However, they have different correlations with the corresponding master images. To analyze the jamming effect on correlation, the correlation map for a pixel array of $256 \times 256$ is obtained. The correlation map before jamming is

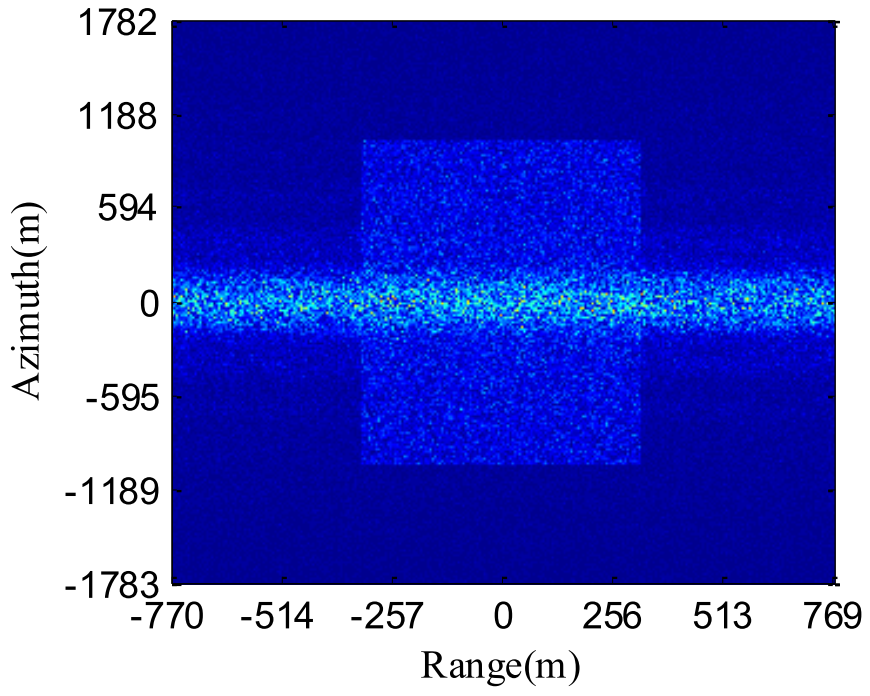

(a)

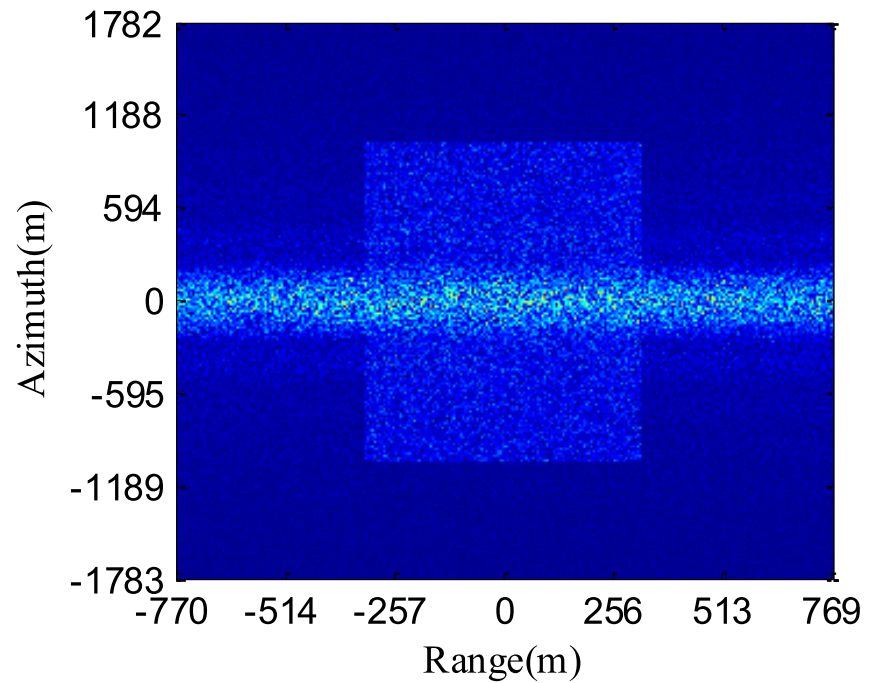

(b)

Fig. 7. Effect on slave images for different jammer locations: (a) non-center located jammer, and (b) center-located jammer.

shown in Fig. 8. It can be observed that the correlation values without jamming are larger than 0.9 and the maximum value approaches to 0.9991 .

When the jammer is not located at the center of the scene, the correlation of the jammed image pair is significantly reduced. When the input jamming to signal ratios are $80 \mathrm{~dB}$ and $100 \mathrm{~dB}$, the correlation maps of the jammed image pair are shown in Fig. 9 (a) and (b), respectively. Clearly, the larger input JSR results in lower correlation of the corresponding pixel pairs. When the jammer is located at the center of the scene, the correlation maps with the input JSR of $80 \mathrm{~dB}$ and $100 \mathrm{~dB}$ are depicted in Fig. 10 (a) and (b). According to (32), the correlation is determined by both the original interferometric phase $\varphi$ and the phase difference $\varphi_{J}$ due to jamming. Moreover, $\varphi_{J}$ approaches to a constant [see (34)] and $\varphi$ is uniformly varied for a flat terrain. Therefore, the correlations of the jammed pixel pairs are shown as varied fringes, which are depicted in Fig. 10(a). In Fig. 10(b), the correlations of the corresponding pixel pairs will be larger, when the larger input JSR is further increased. 


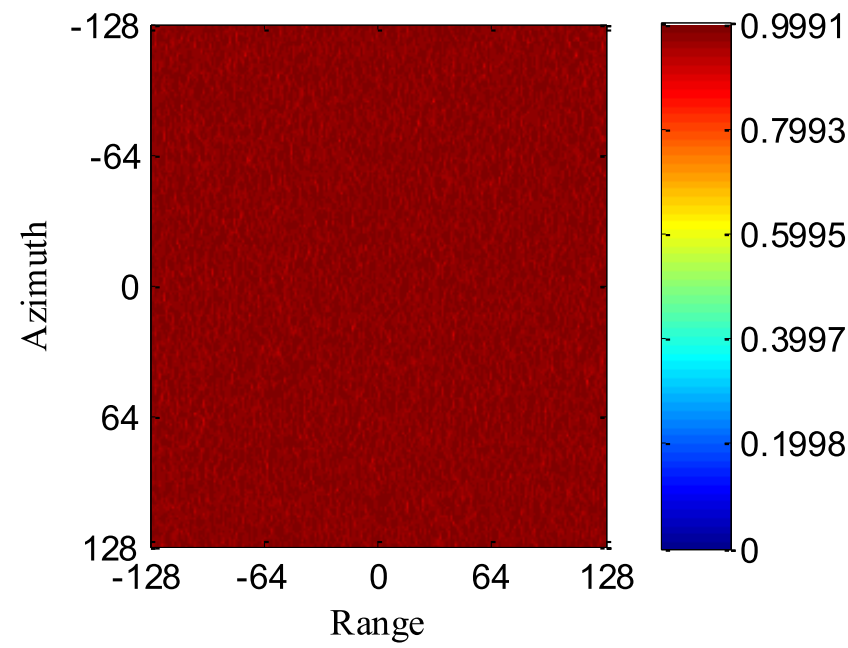

Fig. 8. The correlation of InSAR image pair before jamming.
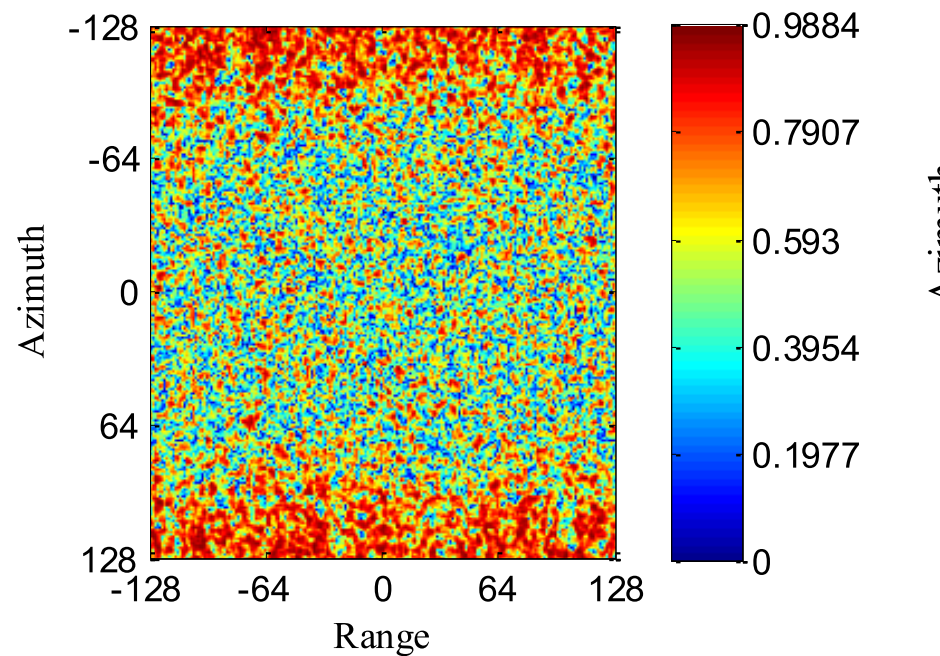

(a)

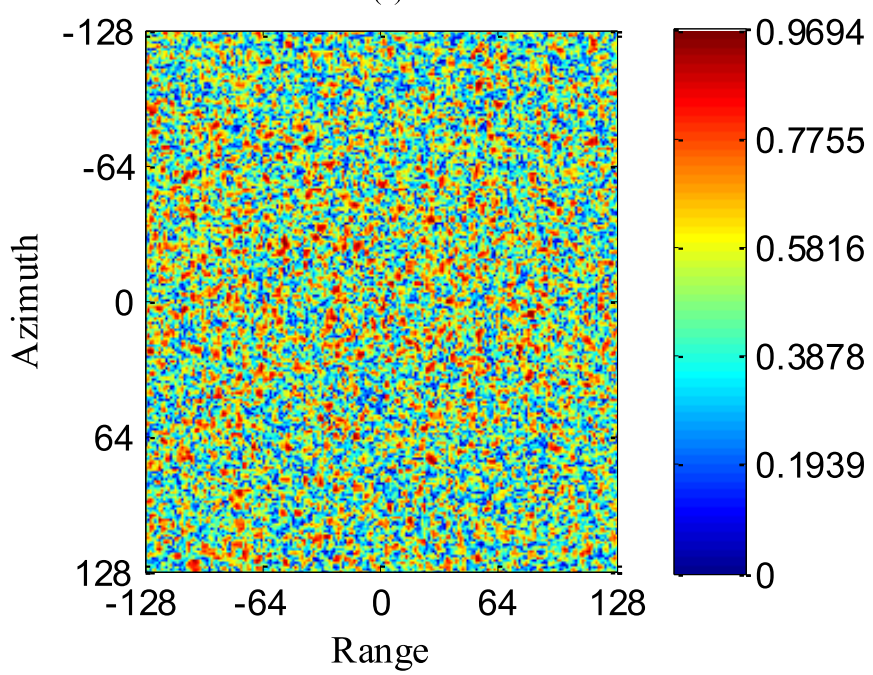

(b)

Fig. 9. Correlation due to the non-center located jammer as the input JSR is $80 \mathrm{~dB}$ (a) and $100 \mathrm{~dB}(\mathrm{~b})$.

The maximum value approaches to 1 , which is larger than those without jamming. Comparing Fig. 9 with Fig. 10, we see that the center located jammer increases the correlation

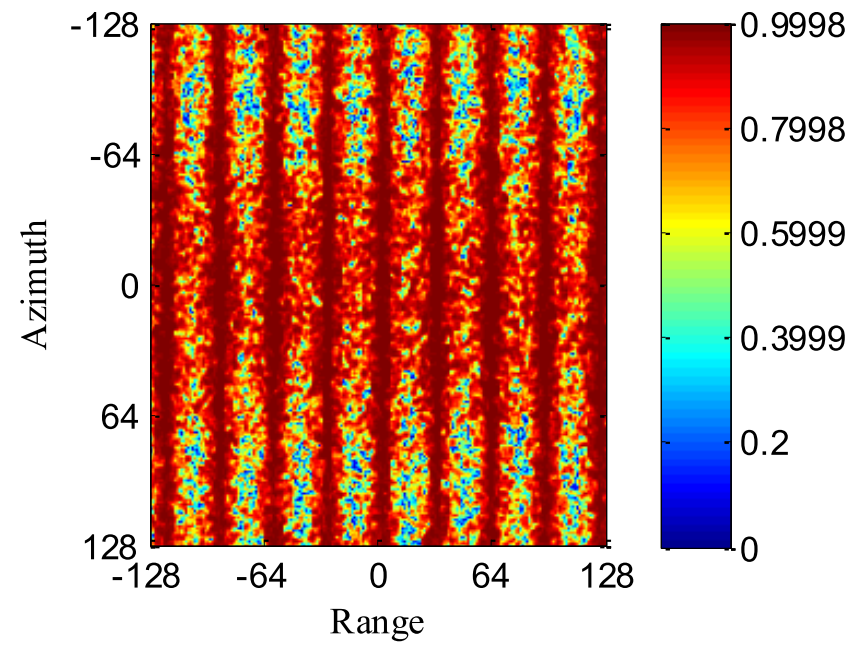

(a)

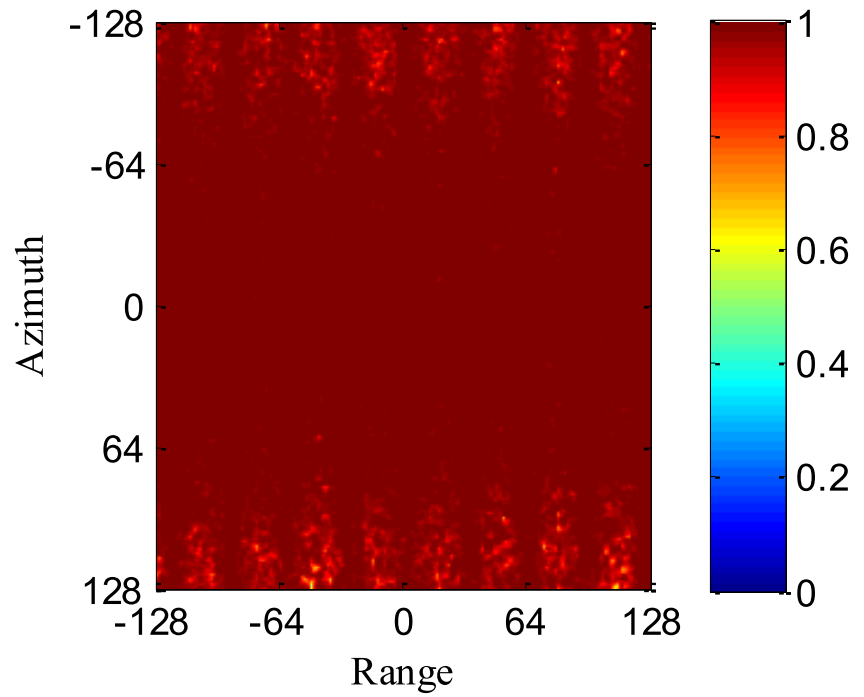

(b)

Fig. 10. Correlation due to the center located jammer as the input JSR is $80 \mathrm{~dB}$ (a) and $100 \mathrm{~dB}(\mathrm{~b})$.

value clearly, while the non-center located one has an opposite effect. As can be seen, the interferences from jammers of different positions cause different jamming effects on correlation. The simulation in this part again demonstrates the effectiveness of the analysis in Section III-A.

\section{Jamming Effect on Interferometric Phase}

To obtain the interferometric phase with interference, the following operations are performed: co-registration and interferometry. Through co-registration and comparing both complex images, the interferometric phases are obtained. For comparison purposes, the original interferometric phase without jamming is shown in Fig. 11(a). The interferometric phase with the phase noises due to the non-center located jammer are presented in Fig. 11(b), while those for the center located jammer are shown in Fig. 11(c). Obviously, the phase noise due to the non-center located jammer changes for different pixel pairs. In Fig. 11(c), the color, representing the phase noise, approaches to red. It means that the values of phase noise in the interferogram are essentially the same 


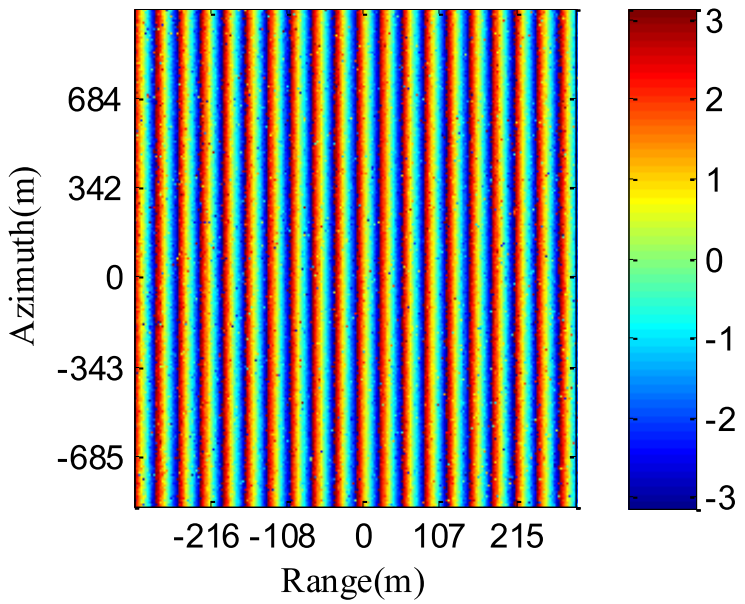

(a)

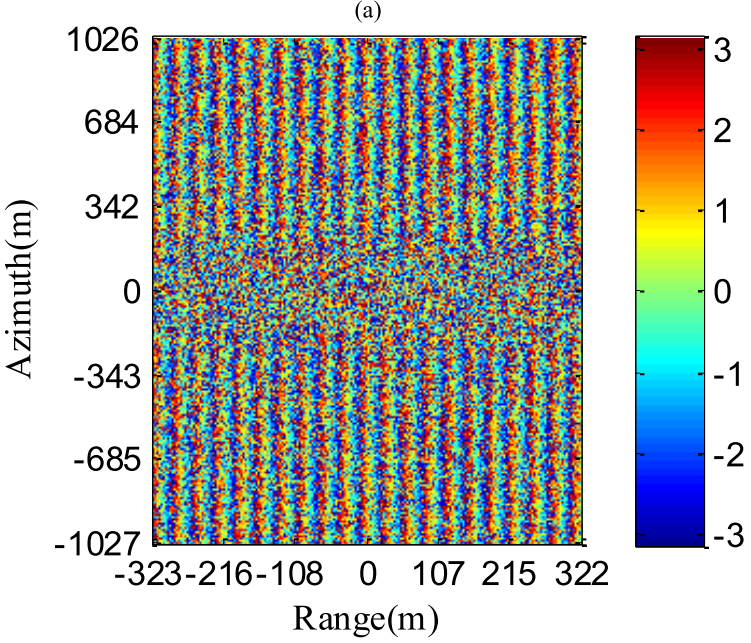

(b)

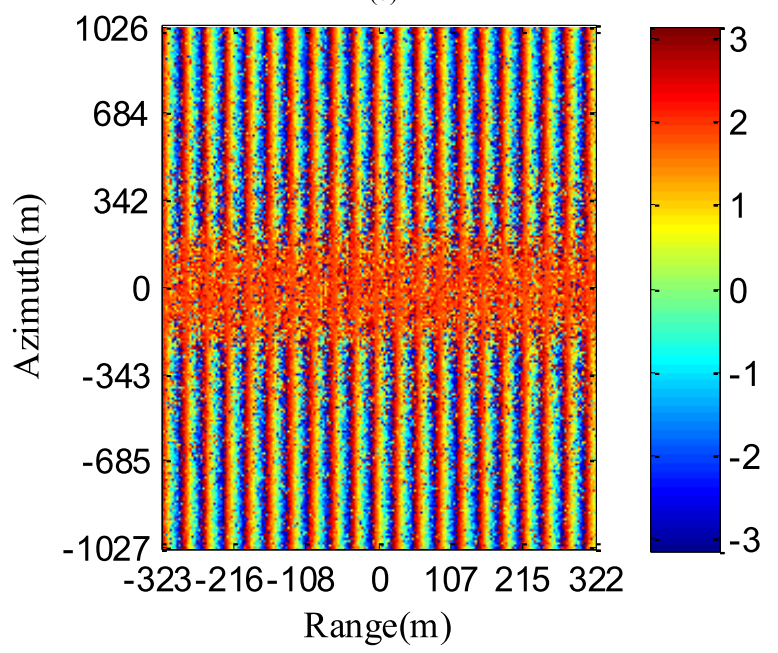

(c)

Fig. 11. Interferometric phases before and after jamming. (a) Original phase. (b) Phase due to non-center located jammer. (c) Phase due to center-located jammer.

with each other, which is corresponding to (31). By comparing Fig. 11 (b) and (c), we see that the interfering effects due to the non-center located jammer are more significant than those caused by the center located one. The phase noises in Fig. 11 (b) are varying, while those in Fig. 11 (c) approach a constant. These results correspond to the analysis in Section III-B.

\section{CONCLuSiON}

In this paper, the effect of interference on the performance of InSAR systems has been analyzed in detail. To study the impact of interference on interferometry, the imaging result of interference is firstly derived. Then, the interfering effects on correlation and interferometric phase are discussed according to different jammer positions. Based on those analyses and supported by simulation results, we can reach the following conclusions:

1) The interference after imaging can be partly compressed along azimuth to some extent and the effect of compression depends on location of the jammer.

2) For the non-center located jammer, an increasing JSR will reduce the correlation of two SAR images and results in more phase errors.

3) For the center located jammer, the correlation of the jammed SAR images is reduced when the power of interference is approximately equal to that of the SAR images. However, a further increase of JSR will have an opposite effect.

4) When the JSR is large enough, the interferometric phase of the corresponding pixel pair approaches a constant with the center located jammer.

All these analyses in our current work are focused on the single-pass InSAR and effective method for mitigating the effect of interference is not investigated. For topics of future research, the impact of interference on dual-pass InSAR will be studied. Displaced Phase Center Antenna (DPCA) can be used to improve the signal to interference ratio of SAR images, while the Differential Interferometric SAR (D-InSAR) technology will be investigated to improve the quality of interferometric phase.

\section{REFERENCES}

[1] H. Breit, T. Fritz, U. Balss, M. Lachaise, A. Niedermeier, and M. Vonavka, "TerraSAR-X SAR processing and products," IEEE Trans. Geosci. Remote Sens., vol. 48, no. 2, pp. 727-740, Feb. 2010.

[2] I. Walterscheid et al., "Bistatic SAR experiments with PAMIR and TerraSAR-X-Setup, processing, and image results," IEEE Trans. Geosci. Remote Sens., vol. 48, no. 8, pp. 3268-3279, Aug. 2010.

[3] R. Romeiser and H. Runge, "Theoretical evaluation of several possible along-track InSAR modes of TerraSAR-X for ocean current measurements," IEEE Trans. Geosci. Remote Sens., vol. 45, no. 1, pp. 21-35, Jan. 2007.

[4] T. Fritz, H. Breit, N. Adam, M. Eineder, and M. Lachaise, "Interferometric SAR processing: From TerraSAR-X to TanDEM-X," in Proc. 7th Eur. Conf. Synth. Aperture Radar, 2008, pp. 1-4.

[5] M. Liao, T. Wang, L. Lu, W. Zhouzhou, and D. Li, "Reconstruction of DEMs from ERS-1/2 tandem data in mountainous area facilitated by SRTM data," IEEE Trans. Geosci. Remote Sens., vol. 45, no. 7, pp. 2325-2335, Jul. 2007.

[6] L. Nengjing and Z. Yiting, "A survey of radar ECM and ECCM," IEEE Trans. Aerosp. Electron. Syst., vol. 31, no. 3, pp. 1110-1120, Jul. 1995.

[7] X. F. Wu, D.-H. Dai, and X.-S. Wang, "Study on SAR jamming measures," in Proc. IET Int. Conf. Radar Syst., Oct. 2007, pp. 1-5.

[8] K. Dumper, P. S. Cooper, A. F. Wons, C. J. Condley, and P. Tully, "Spaceborne synthetic aperture radar and noise jamming," in Proc. IEEE Int. Radar Conf., Edinburgh, U.K., Oct. 1997, pp. 411-417.

[9] F. Zhou, M. Tao, X. Bai, and J. Liu, "Narrow-band interference suppression for SAR based on independent component analysis," IEEE Trans. Geosci. Remote Sens., vol. 51, no. 10, pp. 4952-4960, Oct. 2013.

[10] S. L. Johnston, "Radar electronic counter-countermeasures," IEEE Trans. Aerosp. Electron. Syst., vol. AES-14, no. 1, pp. 109-117, Jan. 1979. 
[11] J. M. Ralston, J. F. Heagy, and R. J. Sullivan, "Environmental noise effects on VHF/UHF UWB SAR," in Proc. Eur. Conf. Synth. Aperture Radar (EUSAR), Friedrichshafen, Germany, May 1998, pp. 141-144.

[12] J. Kim and Y. K. Kwag, "Interference effect analysis from ground based radar in high resolution spaceborne SAR image," in Proc. 3rd Int. Asia-Pacific Conf. Synth. Aperture Radar (APSAR), Sep. 2011, pp. 1-4.

[13] G. Cazzaniga and A. M. Guarnieri, "Removing RF interferences from P-band airplane SAR data," in Proc. IEEE Geosci. Remote Sens. Symp. (IGARSS), vol. 3. Lincoln, NE, USA, May 1996, pp. 1845-1847.

[14] B. Ding, M. S. Xiang, and X. D. Liang, "Analysis of the effect of radio frequency interference on interferometric phase," in Proc. IEEE Geosci. Remote Sens. Symp. (IGARSS), Jul. 2010, pp. 4628-4631.

[15] L. Jia et al., "Analysis on the effects of rebound jamming on InSAR imaging," Electron. Warfare Technol., vol. 27, no. 3, pp. 42-48, May 2012.

[16] Q. Liu, S. Xing, X. Wang, J. Dong, D. Dai, and Y. Li, "The interferometry phase of InSAR coherent jamming with arbitrary waveform modulation," Progr. Electromagn. Res., vol. 124, pp. 101-118, Jan. 2012.

[17] T. Miller, L. Potter, and J. McCorkle, "RFI suppression for ultra wideband radar," IEEE Trans. Aerosp. Electron. Syst., vol. 33, no. 4, pp. 1142-1156, Oct. 1997.

[18] Y. L. Neo, F. H. Wong, and I. G. Cumming, "Processing of azimuthinvariant bistatic SAR data using the range doppler algorithm," IEEE Trans. Geosci. Remote Sens., vol. 46, no. 1, pp. 14-21, Jan. 2008.

[19] H. A. Zebker and J. Villasenor, "Decorrelation in interferometric radar echoes," IEEE Trans. Geosci. Remote Sens., vol. 30, no. 5, pp. 950-959, Sep. 1992.

[20] H. A. Zebker and K. Chen, "Accurate estimation of correlation in InSAR observations," IEEE Geosci. Remote Sens. Lett., vol. 2, no. 2, pp. 124-127, Apr. 2005.

[21] Z. Li, Z. Bao, and Z. Suo, "A joint image coregistration, phase noise suppression, and phase unwrapping method based on subspace projection for multibaseline InSAR systems," IEEE Trans. Geosci. Remote Sens., vol. 45, no. 3, pp. 584-591, Mar. 2007.

[22] C. Maire and M. Datcu, "Earth observation image and DEM information aggregation for realistic 3-D visualization of natural landscapes," IEEE Trans. Geosci. Remote Sens., vol. 43, no. 11, pp. 2676-2683, Nov. 2005.

[23] H. Xu, W. Chen, W. Liu, and S. Li, "Phase statistics for strong scatterers in SAR interferograms," IEEE Geosci. Remote Sens. Lett., vol. 11, no. 11 , pp. 1966-1970, Nov. 2014

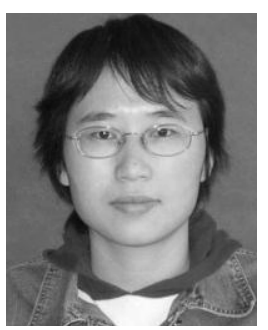

Huaping Xu was born in Shannxi, China, in 1977 She received the B.S. degree in electronics engineering and the $\mathrm{Ph} . \mathrm{D}$. degree in communication and information system from Beihang University (the Beijing University of Aeronautics and Astronautics), Beijing, China, in 1998 and 2003, respectively.

She is currently with the School of Electronic and Information Engineering, Beihang University. Her current research is in SAR, SAR interferometry, differential SAR interferometry, and SAR image processing.

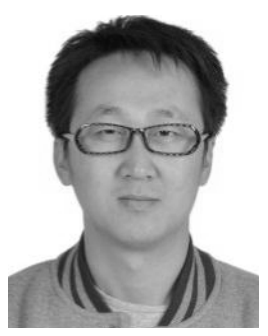

Zhefeng Wu was born in Xinzhou, China, in 1988. He received the M.S. degree in electronics engineering from Beihang University (the Beijing University of Aeronautics and Astronautics), Beijing, China, in 2012, where he is currently pursuing the $\mathrm{Ph} . \mathrm{D}$. degree.

His current research is SAR interferometry and radar countermeasure.

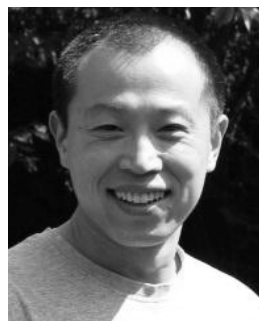

Wei Liu (SM'10) received the B.Sc. and L.L.B degrees from Peking University, China, in 1996 and 1997, respectively, the M.Phil. degree from the University of Hong Kong, in 2001, and the Ph.D. degree from the School of Electronics and Computer Science, University of Southampton, U.K., in 2003. He later held a post-doctoral position with Imperial College London. Since 2005, he has been with the Department of Electronic and Electrical Engineering, The University of Sheffield, U.K., first as a Lecturer, and then a Senior Lecturer. He has authored over 160 journal and conference papers, three book chapters, and a research monograph in wideband beamforming [Wideband Beamforming: Concepts and Techniques (Wiley, 2010)]. His research interests are in sensor array signal processing, blind signal processing, multirate signal processing, and their various applications in radar, sonar, and wireless communications. $\mathrm{He}$ is a member of the Digital Signal Processing Technical Committee of the IEEE Circuits and Systems Society, and an Associate Editor of the IEEE TRANS ACTIONS ON SignaL PROCESSING.

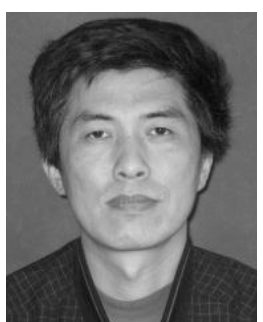

Jingwen Li was born in Qiqihar, China, in 1964. $\mathrm{He}$ received the M.S. and Ph.D. degrees from Beihang University (the Beijing University of Aeronautics and Astronautics), Beijing, China, in 1988 and 1999, respectively.

$\mathrm{He}$ has been a Professor with the School of Electronic and Information Engineering, Beihang University, since 2000 . He is currently the Chair of the Radar Satellite System Simulation Laboratory, Beihang University. His current research interests include SAR image processing, new SAR system design, and SAR GMTI methods.

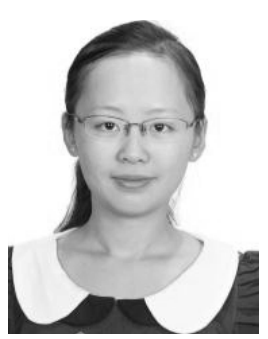

Qingqing Feng was born in Shandong, China, in 1993. She received the bachelor's degree in electronics engineering from Beihang University (the Beijing University of Aeronautics and Astronautics), Beijing, China, in 2015, where she is currently pursuing the M.S. degree.

Her current research is SAR interferometry and SAR image processing. 